\title{
Using a dog's superior olfactory sensitivity to identify Clostridium difficile in stools and patients: proof of principle study
}

\author{
(@) $(1) \Theta$ OPEN ACCESS
}

Marije K Bomers consultant ${ }^{1}$, Michiel A van Agtmael consultant ${ }^{1}$, Hotsche Luik canine trainer and psychologist $^{2}$, Merk C van Veen resident ${ }^{3}$, Christina M J E Vandenbroucke-Grauls professor ${ }^{4}$, Yvo M Smulders professor ${ }^{1}$

${ }^{1}$ Department of Internal Medicine, VU University Medical Centre, PO Box 7057, 1007 MB Amsterdam, Netherlands; ${ }^{2}$ Scent Detection Academy and Research, Animal Behaviour and Cognition, HL\&HONDEN, Edam, Netherlands; ${ }^{3}$ Department of Internal Medicine, St Lucas Andreas Hospital, Amsterdam, Netherlands; ${ }^{4}$ Department of Medical Microbiology and Infection Control, VU University Medical Centre

\begin{abstract}
Objective To investigate whether a dog's superior olfactory sensitivity can be used to detect Clostridium difficile in stool samples and hospital patients.

Design Proof of principle study, using a case-control design.

Setting Two large Dutch teaching hospitals.

Participants A 2 year old beagle trained to identify the smell of $C$ difficile and tested on 300 patients ( 30 with $C$ difficile infection and 270 controls).

Intervention The dog was guided along the wards by its trainer, who was blinded to the participants' infection status. Each detection round concerned 10 patients (one case and nine controls). The dog was trained to sit or lie down when $C$ difficile was detected.
\end{abstract}

Main outcome measures Sensitivity and specificity for detection of $C$ difficile in stool samples and in patients.
Results The dog's sensitivity and specificity for identifying $C$ difficile in stool samples were both $100 \%$ (95\% confidence interval $91 \%$ to $100 \%$ ). During the detection rounds, the dog correctly identified 25 of the 30 cases (sensitivity $83 \%, 65 \%$ to $94 \%$ ) and 265 of the 270 controls (specificity $98 \%$, $95 \%$ to $99 \%$ ).

Conclusion $\mathrm{A}$ trained dog was able to detect $C$ difficile with high estimated sensitivity and specificity, both in stool samples and in hospital patients infected with $C$ difficile.

\section{Introduction}

Clostridium difficile infection is common, particularly in people in healthcare facilities who have received antimicrobials. $C$ difficile causes toxin mediated intestinal disease, with symptoms ranging from mild diarrhoea to severe pseudomembranous colitis and toxic megacolon. The bacterium can be transmitted through

\section{Correspondence to: M K Bomers m.bomers@vumc.nl}

Video on bmj.com (see also http://bmj.com/video)

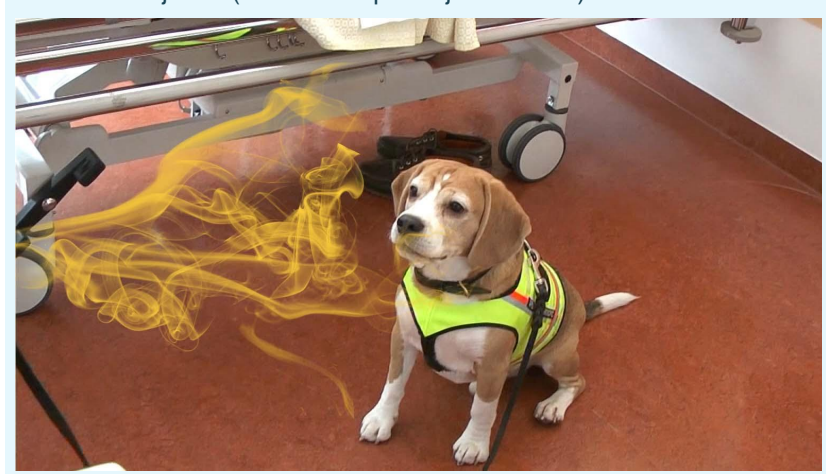

Cliff has been trained to sniff out the bacteria clostridium difficile 
either personal contact or the environment. ${ }^{1}$ Since 2000 more frequent and severe disease has emerged and large outbreaks in hospitals have necessitated ward closures and extensive infection control measures. ${ }^{2-4}$ Infection rates seem to be higher in North America than in Europe. ${ }^{35}$ In the Netherlands the incidence of nosocomial $C$ difficile infection is comparable to that of other European countries (mean incidence 17.5-23/10 000 admissions $)^{67}$; the mean incidence in the United Kingdom is $50 / 10000$ admissions. $^{6}$

Early and rapid identification of $C$ difficile infection is important for the initiation of infection control measures and treatment to prevent transmission. ${ }^{8}$ Several combinations of tests are used to diagnose cases. The traditional standard is by cytotoxin assay, which if $C$ difficile toxins are present shows cytotoxicity of faecal eluate on mammalian cells. This technique requires cell cultures, however, and results take at least 1-2 days. ${ }^{910}$ Culture on selective media is very sensitive but also time consuming and it lacks specificity because of possible carriage of non-toxigenic isolates. Cultured strains can subsequently be tested for the production of toxins, in which case this is referred to as a toxigenic culture. ${ }^{4}{ }^{10}$ Easy and rapid enzyme immunoassays to detect $C$ difficile toxins or antigens are often used, despite their limited sensitivity or specificity. ${ }^{10}{ }^{11}$ More recently, several nucleic acid amplification tests have been developed that have a high diagnostic accuracy and short turnaround time, although these tests are expensive and require specialised equipment and expertise..$^{4-12}$

In daily practice, several factors delay the identification of $C$ difficile infections. These include doctor's delay (for example, the doctor does not consider the possibility of a $C$ difficile infection, or decides to wait and see if the symptoms pass), inefficient sampling, and time required to process samples in the laboratory. ${ }^{13}{ }^{14}$ As a result the mean time from onset of symptoms to start of treatment in studies ranges from 2.8 to 7.7 days. ${ }^{13} 14$ This can result in spread of $C$ difficile infection by delaying appropriate infection control measures such as transferring patients to a single room. Screening all hospital patients at regular intervals could theoretically prevent delays in diagnosis but this is costly and impractical.

In the 1970s $C$ difficile was identified as the cause of pseudomembranous colitis. ${ }^{15}{ }^{16}$ Since then, $C$ difficile associated diarrhoea has often been described as having a characteristic smell. ${ }^{17}$ Sensitivity and specificity of the odiferous detection of $C$ difficile by nursing staff are $55-82 \%$ and $77-83 \%$, respectively. ${ }^{18}{ }^{19}$ Dogs have a far superior sense of smell, however, which is thought to exceed that of humans by a factor of $100 .^{20} \mathrm{We}$ hypothesised that it may be possible for a dog to be trained to recognise the presence of $C$ difficile in stool samples, or even in patients. If so, this could prove a valuable screening tool for $C$ difficile infections in healthcare facilities.

\section{Methods}

\section{The training process}

The dog used in this study was a 2 year old male beagle (fig $1 \Downarrow$ ). A professional detection dog instructor (HL) trained the $\operatorname{dog}$ to identify $C$ difficile in stool samples and, if this proved possible, in patients. A reward based training method was used in which the correct behaviour was reinforced, for instance by providing a treat. The dog was taught to sit or lie down if a specific scent was detected. He had not received previous training in detection.

Training began by introducing the dog to the specific odour of toxigenic $C$ difficile strains on culture plates. Wooden sticks were placed over the sample to absorb the scent emanating from the culture plates (often described as resembling horse manure ${ }^{9}$ ). Initially we left the sticks in this position overnight, but eventually the exposure time was shortened to around five minutes to reduce the strength of the scent. Early recognition of the scent was achieved by using simple search and find games, which were gradually replaced by exercises of increasing difficulty, for instance by using sticks with a fainter smell (that had not been exposed to the scent as long). Also, the scent was presented to the dog on materials other than the sticks, such as paper, fabric, and metal, and in different environments such as a kitchen, forest, or petrol station to vary background odours.

The next step of training concerned discrimination between stool samples that were positive for toxin producing $C$ difficile strains from those that were negative. Again, rather than the dog having direct contact with the stool sample, we presented the scent in various forms (absorbed on a wooden stick or on fabric, sample contained in a plastic vial, etc).

After two months' training we formally tested the diagnostic accuracy of the dog on stool samples. Finally, we explored the dog's abilities to detect $C$ difficile infection in patients admitted to hospital.

\section{Samples and participants $C$ difficile on culture plates}

For training purposes we used clinical isolates of toxigenic $C$ difficile strains, cultured on standard media under anaerobic conditions.

\section{Stool samples}

We used stool samples that were sent to the microbiology laboratory to test for $C$ difficile and other infectious causes of diarrhoea. Samples were considered positive for $C$ difficile if toxin was detected by enzyme immunoassay (VIDAS Clostridium difficile A \& B; BioMérieux, France) and culture revealed a toxigenic strain of $C$ difficile. Negative stool samples were those that gave negative results in both tests. We excluded samples with inconsistent results (for example, negative result for toxin by enzyme immunoassay but positive culture result, or an undetermined toxin enzyme immunoassay value).

\section{Selection of participants}

We further explored the dog's ability to detect $C$ difficile infection on the wards of two hospitals in Amsterdam: VU University medical centre (a tertiary clinical care centre) and St Lucas Andreas Hospital (a large community hospital).

Between September 2010 and May 2011, we screened for inclusion consecutive patients who had a positive result for toxin by enzyme immunoassay in their stool sample. We aimed to include 30 patients in total. Both hospitals use an enzyme immunoassay plus a toxigenic culture to diagnose $C$ difficile infection; however, the community hospital uses an enzyme immunoassay by a different manufacturer (ImmunoCard Toxins $\mathrm{A} \& \mathrm{~B}$; Meridian Bioscience, Cincinnati, $\mathrm{OH}$ ).

Eligible cases had symptoms of diarrhoea and both a positive result for toxin by enzyme immunoassay and a toxigenic $C$ difficile strain detected by culture (in a sample taken less than seven days before the detection round). We defined diarrhoea as three or more loose or watery stool passages a day. We excluded children and adults on intensive care units or haematology wards. Patients with a first relapse after completing treatment for a previous $C$ difficile infection were eligible but not those with subsequent relapses. Because the availability of 
the dog and trainer could not be guaranteed every weekend we excluded patients with positive test results during the weekend.

For each case we approached nine control patients. These patients were on the same ward as and in close proximity to the index patient. Control patients did not have diarrhoea, or had diarrhoea but a negative result for $C$ difficile toxin by enzyme immunoassay and culture (in a sample taken less than seven days previously). All participants ( $\mathrm{n}=300 ; 30$ cases plus 270 controls) gave informed consent.

\section{Canine testing experiment}

\section{Diagnostic accuracy for detecting $C$ difficile in stool samples}

We formally tested the dog's diagnostic accuracy on 50 stool samples with known positivity for $C$ difficile and 50 with known negativity after completion of a two month practice period. To avoid the possibility that the dog simply recognised the odour of the sample rather than the odour being associated with the presence of $C$ difficile, we did not use the samples that had been used during training. The scent of each sample was again absorbed onto different materials, which were then repeatedly (10 times) presented to the dog in different environments and at different concentrations. We considered a result to be positive (or negative) when it consistently provoked the same positive (or negative) response. If a sample provoked a mixed response ( $\leq 8 / 10$ consistency: for example, eight positive responses and two negative ones), we classified it as an inconclusive result.

\section{Diagnostic accuracy for detecting $C$ difficile in patients}

Next we evaluated the dog's detection abilities in patients on the wards of the two hospitals. We prospectively included 30 consecutive patients with $C$ difficile infection and 270 control patients. For each case and corresponding nine controls on the ward we organised a detection round as soon as possible, preferably before starting treatment or within 36 hours. During this round the dog, trainer, and a member of the research team simply walked past the beds of the 10 participants. The trainer classified the dog's response as either positive (dog sat down), inconclusive (dog showed excitement, took extra time without actually sitting down), or negative (dog showed no particular interest). We assumed the dog would be able to identify the patient with a $C$ difficile infection by smelling the air surrounding the patient (independent of a patient's bowel movements or personal hygiene), so physical contact was unnecessary and avoided. If there was any doubt, the round was repeated once. The trainer was not aware of which patients had C difficile infection.

\section{Statistical analyses}

In the primary analyses, we interpreted inconclusive responses as negative ones. Secondary analysis was done with these responses interpreted as positive. We calculated $95 \%$ confidence intervals using an approximation (according to the efficient score method, corrected for continuity). ${ }^{21}$

\section{Safety precautions}

We consulted the hospital's infection control committee to discuss the potential hazards of allowing a dog in the hospital. In accordance with recent guidelines, special attention was given to hand hygiene, making sure that staff and patients washed their hands both before and after contact with the dog. ${ }^{22}$ During detection rounds, the dog had no physical contact with patients, and contact with the environment (for example, bed, chair) was avoided as much as possible. The dog was not allowed in food preparation areas or on neonatal, haematology, or intensive care wards.

The dog receives a health evaluation by a licensed veterinarian four times a year. He is not fed raw meat. He is trained solely for the purpose of recognising $C$ difficile. When at work, he neither barks nor shows aggression, is easily recognised by his outfit (fig 1), and is always on a leash.

\section{Results \\ Diagnostic accuracy for detecting $C$ difficile in stool samples}

The dog was presented with a total of 100 stool samples: 50 were positive for $C$ difficile and 50 were negative. The dog gave a positive response to all 50 positive samples and a negative response to 47 of the 50 negative samples, with the remaining three negative samples recorded as an inconclusive response. In the primary analysis (interpreting inconclusive responses as negative responses), sensitivity and specificity were both $100 \%$ ( $95 \%$ confidence interval $91 \%$ to $100 \%$ ). If an inconclusive response was considered as a positive result (secondary analysis), the dog's sensitivity and specificity were $100 \%$ (91\% to $100 \%$ ) and $94 \%$ ( $83 \%$ to $98 \%$ ), respectively.

\section{Patients' characteristics}

The supplementary video illustrates how the detection rounds were carried out. One round took place on a paediatric ward but the children became excitable by having an animal on the ward and distracted the dog. For this reason the round was not included and paediatric wards were excluded from the study.

Thirty patients with $C$ difficile infection and 270 control patients were included in the study. Table $1 \Downarrow$ shows the characteristics of those patients. On the day of the detection round all 30 cases had diarrhoea compared with $16(6 \%)$ of the controls. Stool samples from 35 controls (13\%) were tested for $C$ difficile on clinical grounds (presence of diarrhoea) in the week leading up to the detection round; these all gave negative results, although a non-toxigenic $C$ difficile strain was cultured from two controls. All but three cases formally met the definition for $C$ difficile infection. The first patient had symptoms of the infection and a positive result for toxin by enzyme immunoassay, but a culture was mistakenly not done. The second patient had symptoms and a positive result for toxin by enzyme immunoassay but an initial negative culture result, which turned out to be positive when repeated. The third patient had a relapse with recurring symptoms and a positive toxigenic culture result but negative result for toxin by enzyme immunoassay.

\section{Diagnostic accuracy for detecting $C$ difficile in patients}

Figure $2 \Downarrow$ illustrates the diagnostic accuracy of the dog. An inconclusive response was recorded in seven participants: three cases and four controls. In the primary analysis (interpreting inconclusive responses as negative results), the dog correctly identified $25 / 30$ cases (sensitivity $83 \%, 95 \%$ confidence interval $65 \%$ to $94 \%$ ) and $265 / 270$ controls (specificity $98 \%, 95 \%$ to $99 \%$ ). If inconclusive responses were considered as positive results (secondary analysis), the dog correctly identified 28 cases (sensitivity $93 \%, 76 \%$ to $99 \%$ ) and 261 controls (specificity $97 \%, 94 \%$ to $98 \%$ ).

Table $2 \Downarrow$ provides information on the occasions that the dog and the laboratory gave discrepant results (inconclusive dog 
responses, false positives, and false negatives). In some instances the dog was clearly distracted by unrelated stimuli (for instance, by being offered a treat). Other cases were less clear and it cannot be ruled out that the dog responded to diarrhoea that was not caused by $C$ difficile (for example, control 7, table 2 ) or asymptomatic carriage of a non-toxigenic strain (for example, control 8, table 2). Of all 16 participants with diarrhoea not related to $C$ difficile infection, the dog gave a negative response in 13 controls and an inconclusive response in three controls.

\section{Discussion}

It is feasible to use a dog to detect Clostridium difficile in stool samples and in patients. The dog's diagnostic accuracy with stool samples suggests that immediate identification of $C$ difficile is possible. Moreover, our data suggest that the same may be true for the rapid diagnosis of $C$ difficile infection on clinical wards. For the purposes of detection the dog did not need a stool sample or physical contact with patients. It would seem dogs can detect $C$ difficile in the air surrounding patients. In addition, dogs are quick and efficient: patients in a hospital ward can be screened for the presence of $C$ difficile infection in less than 10 minutes.

This is the first report of animal assisted detection of $C$ difficile. There have been several studies and anecdotal reports on olfactory detection in medicine, mostly using dogs-for example, to detect malignancies of bladder, lung, breast, skin, prostate, ovary, and colon. ${ }^{23-29}$ In nearly all the studies, however, the animals were exposed to biological samples obtained from patients and not to the patients themselves.

\section{Limitations of the study}

Our study has several limitations. The small number of patients with $C$ difficile infection limits the precision with which we can establish the dog's sensitivity and specificity. The design of the study consistently included one case per round of 10 patients. Anticipation of a single positive result could have influenced the trainer's behaviour, thereby unintentionally influencing the dog's response. ${ }^{30}$ Furthermore, two thirds of the cases had been moved to a single room (to control transmission) when the dog arrived, and occupancy of a single room might again have influenced the trainer and therefore the dog's response. ${ }^{30}$

In this study, culture was not routinely carried out on controls to screen for asymptomatic carriage of toxigenic and non-toxigenic $C$ difficile. This is a limitation as consequently we do not know the percentage of asymptomatic $C$ difficile carriers in our population and how the dog responded to them. Asymptomatic carriage of both toxigenic and non-toxigenic strains occurs in up to $18-30 \%$ of patients in hospital. ${ }^{31-34}$ This argues against a positive response by the dog. As the clinical relevance of detecting $C$ difficile infection rather than carriage is far greater (both for the patient and for the prevention of transmission ${ }^{131}$ ), this was the focus of our study.

Another concern is that the results are not easily generalisable because we used only one dog and one trainer. It could be that the findings would be less convincing for another dog or trainer. Although unlikely, we cannot rule out the possibility that our first and only experience was with an exceptional dog-trainer combination. Should more dogs become available in the future for detection of $C$ difficile infection, trained animals would need an individual assessment of performance and regular practice to maintain their skills. A second limitation of using an animal as a diagnostic tool is that, as with humans, behaviour is not fully predictable. The dog's reaction to other stimuli (for example, children's play, being beckoned, being offered a treat) illustrates that, despite a high level of training, dogs are still prone to distraction.

Another limitation is that we trained the dog in the hospital setting. Outside the research protocol we visited a few patients with $C$ difficile infection on long term care facility wards. These cases spent much of the day in a shared living room and not in their beds. This proved more difficult for the dog. We hypothesise that in the hospital setting the bed is a strong source of smell because the patients are often bedridden and the mattress is more likely absorb odours. Patients in the community setting are often less confined to their room and bed. This could make odours more diffuse and more difficult to pinpoint. Also, the dog may have been conditioned to respond to the bacterium when a patient was in a hospital room (usually in bed). This may mean that detection dogs are less suitable for other settings such as nursing homes, at least without additional training.

The use of dogs in hospitals might pose a risk to the dogs themselves, hospital staff, and patients. Dogs can be carriers of $C$ difficile strains and other pathogens. Similar to hospital staff, the dog could be a source of transmission. This risk could be minimised by using strict preventive measures such as avoiding physical contact with patients and their surroundings.

\section{Unanswered questions}

Unanswered questions remain, such as what does the dog actually smell-is it a certain quantity of bacteria, toxins, or other bacterial products? How does the dog respond to stool samples that are negative for toxin by enzyme immunoassay but positive by toxigenic culture, cytotoxicity assay, or nucleic acid amplification tests? And how does the dog respond to patients early in the course of the infection, or those with asymptomatic carriage of toxigenic and non-toxigenic strains? Does a positive response by the dog to a patient without symptoms predict disease? Can a persistent positive response by the dog after symptoms have cleared predict relapse, as suggested by the response to one participant (control 12, table 2)? Will the dog perform equally well in a high incidence setting - that is, during an outbreak, when several patients in one room could be affected? We intend to deal with these situations in future studies.

How could a dog that detects $C$ difficile infection be used in daily practice? With regular surveillance rounds (for instance to screen all wards in a hospital with a high incidence of infection several times a week, a "pet scan") $C$ difficile might be detected earlier. Early detection could overcome common diagnostic delays (lack of clinical suspicion, delays in sampling stool, and laboratory procedures) and lead to prompt hygienic measures and treatment. However, further studies will clearly have to examine whether surveillance can actually limit transmission and reduce the incidence of infection. For example, surveillance is principally different from the type of case directed diagnosis in this study design, because the dog cannot immediately receive a reward after a positive identification, potentially extinguishing the trained alert.

In conclusion, in this proof of principle study a trained dog was able to detect $C$ difficile with high estimated sensitivity and specificity, both in stool samples and in infected patients in a hospital setting. This finding could have great potential for screening for $C$ difficile infection in healthcare facilities and thus contribute to the control and prevention of outbreaks.

Contributors: MKB, MvA, CvdB-G, and YMS developed the concept and outlined the methods. $\mathrm{HL}$ trained the dog. MKB, $\mathrm{HL}$, and MvV carried out the detection rounds and collected the data. MKB analysed the data 


\section{What is already known on this topic}

Early and rapid identification of Clostridium difficile infection (CDI) is important to prevent transmission by initiating adequate isolation measures and treatment

Studies have, however, shown that the mean time between the onset of symptoms and treatment is about 3-8 days

Despite the availability of a wide range of diagnostic tests, $\mathrm{CDI}$ is still a widespread healthcare related infection

\section{What this study adds}

A dog can be trained to identify $C$ difficile with high estimated sensitivity and specificity, both in stool samples and in hospital patients with CDI

The potential of using a dog for detection is the ability to screen hospital wards for infected patients

Such screening could overcome common delays in diagnosis and thus help to control and prevent CDI outbreaks

and drafted the paper. MvA, CvdB-G, and YMS provided conceptual and technical guidance. All authors critically appraised the paper, revised where appropriate and approved the final version of the manuscript. MKB and YMS are the guarantors.

Competing interests: All authors have completed the ICMJE uniform disclosure form at www.icmje.org/coi_disclosure.pdf (available on request from the corresponding author) and declare: $\mathrm{HL}$ is the owner and chairman of the Animal Behaviour and Cognition, Scent Detection Research and Academy HL\&HONDEN, Edam, Netherlands; otherwise no support from any organisation for the submitted work; no financial relationships with any organisations that might have an interest in the submitted work in the previous three years; no other relationships or activities that could appear to have influenced the submitted work.

Ethical approval: The research protocol was approved by the institutional review boards of both hospitals.

Data sharing: No additional data available.

Samore MH, Venkataraman L, DeGirolami PC, Arbeit RD, Karchmer AW. Clinical and molecular epidemiology of sporadic and clustered cases of nosocomial Clostridium difficile diarrhea. Am J Med 1996;100:32-40.

2 Loo VG, Poirier L, Miller MA, Oughton M, Libman MD, Michaud S, et al. A predominantly clonal multi-institutional outbreak of Clostridium difficile-associated diarrhea with high morbidity and mortality. N Engl J Med 2005;353:2442-9.

3 Freeman J, Bauer MP, Baines SD, Corver J, Fawley WN, Goorhuis B, et al. The changing epidemiology of Clostridium difficile infections. Clin Microbiol Rev 2010;23:529-49.

4 Rupnik M, Wilcox MH, Gerding DN. Clostridium difficile infection: new developments in epidemiology and pathogenesis. Nat Rev Microbiol 2009;7:526-36.

5 Kuijper EJ, Coignard B, Tull P. Emergence of Clostridium difficile-associated disease in North America and Europe. Clin Microbiol Infect 2006;12(Suppl 6):2-18.

6 Bauer MP, Notermans DW, van Benthem BH, Brazier JS, Wilcox MH, Rupnik M, et al. Clostridium difficile infection in Europe: a hospital-based survey. Lancet 2011;377:63-73. Hensgens MP, Goorhuis A, van Kinschot CM, Crobach MJ, Harmanus C, Kuijper EJ. Clostridium difficile infection in an endemic setting in the Netherlands. Eur J Clin Microbiol Infect Dis 2011;30:587-93.

8 Muto CA, Blank MK, Marsh JW, Vergis EN, O'Leary MM, Shutt KA, et al. Control of an outbreak of infection with the hypervirulent Clostridium difficile BI strain in a university hospital using a comprehensive "bundle" approach. Clin Infect Dis 2007;45:1266-73.

9 Delmee M. Laboratory diagnosis of Clostridium difficile disease. Clin Microbiol Infect 2001;7:411-6.

10 Kufelnicka AM, Kirn TJ. Effective utilization of evolving methods for the laboratory diagnosis of Clostridium difficile infection. Clin Infect Dis 2011:52:1451-7.

11 Crobach MJ, Dekkers OM, Wilcox MH, Kuijper EJ. European Society of Clinical Microbiology and Infectious Diseases (ESCMID): data review and recommendations for diagnosing Clostridium difficile-infection (CDI). Clin Microbiol Infect 2009;15:1053-66.

12 Karre T, Sloan L, Patel R, Mandrekar J, Rosenblatt J. Comparison of two commercial molecular assays to a laboratory developed molecular assay for diagnosis of Clostridium difficile infection. J Clin Microbiol 2011:49:725-7.

13 Frenz MB, McIntyre AS. Reducing delays in the diagnosis and treatment of Clostridium difficile diarrhoea. Q J Med 2003;96:579-82.

14 Scheurer D. Diagnostic and treatment delays in recurrent Clostridium difficile-associated disease. J Hosp Med 2008;3:156-9.
15 Bartlett JG, Onderdonk AB, Cisneros RL, Kasper DL. Clindamycin-associated colitis due to a toxin-producing species of Clostridium in hamsters. $J$ Infect Dis 1977;136:701-5.

16 George RH, Symonds JM, Dimock F, Brown JD, Arabi Y, Shinagawa N, et al. Identification of Clostridium difficile as a cause of pseudomembranous colitis. BMJ 1978;1:695.

17 Bartlett JG, Gerding DN. Clinical recognition and diagnosis of Clostridium difficile infection. Clin Infect Dis 2008;46(Suppl 1):S12-8.

18 Burdette SD, Bernstein JM. Does the nose know? The odiferous diagnosis of Clostridium difficile-associated diarrhea. Clin Infect Dis 2007:44:1142.

19 Johansen A, Vasishta S, Edison P, Hosein I. Clostridium difficile associated diarrhoea: how good are nurses at identifying the disease? Age Ageing 2002;31:487-8.

20 Issel-Tarver L, Rine J. The evolution of mammalian olfactory receptor genes. Genetics 1997;145:185-95.

21 Newcombe RG. Two-sided confidence intervals for the single proportion: comparison of seven methods. Stat Med 1998;17:857-72.

22 Lefebvre SL, Golab GC, Christensen E, Castrodale L, Aureden K, Bialachowski A, et al. Guidelines for animal-assisted interventions in health care facilities. Am J Infect Control 2008;36:78-85.

23 Willis CM, Church SM, Guest CM, Cook WA, McCarthy N, Bransbury AJ, et al. Olfactory detection of human bladder cancer by dogs: proof of principle study. BMJ 2004;329:712.

24 McCulloch M, Jezierski T, Broffman M, Hubbard A, Turner K, Janecki T. Diagnostic accuracy of canine scent detection in early- and late-stage lung and breast cancers. Integr Cancer Ther 2006;5:30-9.

25 Ehmann R, Boedeker E, Friedrich U, Sagert J, Dippon J, Friedel G, et al. Canine scent detection in the diagnosis of lung cancer: revisiting a puzzling phenomenon. Eur Respir J 2012;39:669-76.

26 Sonoda H, Kohnoe S, Yamazato T, Satoh Y, Morizono G, Shikata K, et al. Colorectal cancer screening with odour material by canine scent detection. Gut 2011:60:814-9.

27 Horvath G, Andersson H, Paulsson G. Characteristic odour in the blood reveals ovarian carcinoma. BMC Cancer 2010;10:643.

28 Cornu JN, Cancel-Tassin G, Ondet V, Girardet C, Cussenot O. Olfactory detection of prostate cancer by dogs sniffing urine: a step forward in early diagnosis. Eur Urol 2011;59:197-201.

29 Williams H, Pembroke A. Sniffer dogs in the melanoma clinic? Lancet 1989;1:734.

30 Lit L, Schweitzer JB, Oberbauer AM. Handler beliefs affect scent detection dog outcomes. Anim Cogn 2011;14:387-94.

31 McFarland LV, Mulligan ME, Kwok RY, Stamm WE. Nosocomial acquisition of Clostridium difficile infection. N Engl J Med 1989;320:204-10.

32 Samore MH, DeGirolami PC, Tlucko A, Lichtenberg DA, Melvin ZA, Karchmer AW. Clostridium difficile colonization and diarrhea at a tertiary care hospital. Clin Infect Dis 1994:18:181-7.

33 Clabots CR, Johnson S, Olson MM, Peterson LR, Gerding DN. Acquisition of Clostridium difficile by hospitalized patients: evidence for colonized new admissions as a source of infection. J Infect Dis 1992;166:561-7.

34 Shim JK, Johnson S, Samore MH, Bliss DZ, Gerding DN. Primary symptomless colonisation by Clostridium difficile and decreased risk of subsequent diarrhoea. Lancet 1998:351:633-6.

Accepted: 24 September 2012

\section{Cite this as: BMJ 2012;345:e7396}

This is an open-access article distributed under the terms of the Creative Commons Attribution Non-commercial License, which permits use, distribution, and reproduction in any medium, provided the original work is properly cited, the use is non commercial and is otherwise in compliance with the license. See: http://creativecommons.org/licenses/bync/2.0/ and http://creativecommons.org/licenses/by-nc/2.0/legalcode. 


\section{Tables}

Table 1/ Characteristics of patients with Clostridium difficile infection and controls. Values are numbers (percentages) unless stated otherwise

\begin{tabular}{|c|c|c|c|}
\hline Characteristics & Controls $(n=270)$ & Cases $(n=30)$ & Total $(n=300)$ \\
\hline Men & $152(56)$ & $15(50)$ & $167(57)$ \\
\hline Median (interquartile range) age (years) & $65(54-78)$ & $68(51-75)$ & $65(54-78)$ \\
\hline \multicolumn{4}{|l|}{ Ward type: } \\
\hline Medical & $165(61)$ & $19(63)$ & $184(61)$ \\
\hline Surgical & $105(39)$ & $11(37)$ & $116(39)$ \\
\hline Diarrhoea on day of detection round ${ }^{*}$ & $16(6)$ & $30(100)$ & $46(15)$ \\
\hline \multicolumn{4}{|l|}{ Clinical characteristics: } \\
\hline No test done and no diarrhoea symptoms $\dagger$ & $235(87)$ & $0(0)$ & - \\
\hline No infection, confirmed by negative test result ${ }^{\star} \dagger$ & $35(13)$ & $0(0)$ & - \\
\hline Infection, confirmed by diarrhoea and positive test result + & $0(0)$ & $30(100)$ & - \\
\hline Treatment for $>36$ hours on day of detection round & $0(0)$ & $3(10)$ & - \\
\hline
\end{tabular}

*All controls with diarrhoea on day of detection round underwent diagnostic testing for $C$ difficile infection and are included in group with no infection and confirmed by negative test result.

$\dagger C$ difficile toxin enzyme immunoassay and culture done on stool sample in seven days before detection round. 


\begin{tabular}{|c|c|c|c|c|}
\hline Variables & Dog response & Laboratory tests* & $\begin{array}{l}\text { Diarrhoea on day of } \\
\text { detection round }\end{array}$ & Comment \\
\hline \multicolumn{5}{|c|}{ Inconclusive dog response: } \\
\hline Case 1 & Inconclusive & Positive result & Yes & Dog appeared distracted by plastic cup on floor \\
\hline Control 2 & Inconclusive & Negative result & Yes & $\begin{array}{l}\text { During this round there was a strong chlorine smell in several rooms, from } \\
\text { disinfection, which could have influenced the dog's response. Tests were } \\
\text { done on participants with an inconclusive response in this round }\end{array}$ \\
\hline Case 3 & Inconclusive & Positive result & Yes & Chlorine round, see comment for control 2 \\
\hline Control 4 & Inconclusive & Not done & No & $\begin{array}{l}\text { Case } 5 \text { had just changed beds; the dog seemed to have difficulty choosing } \\
\text { between two neighbouring patients (control } 4 \text { and case } 5 \text { ) and the third } \\
\text { (empty) bed across the room; he sat in the middle }\end{array}$ \\
\hline Case 5 & Inconclusive & Positive result & Yes & See comment for control 4 \\
\hline Control 6 & Inconclusive & Negative result & Yes & $\begin{array}{l}\text { On the ward was a patient with Clostridium difficile infection who refused } \\
\text { to participate in the study. The dog was not allowed to enter the patient's } \\
\text { room; however, the dog was excited and tried to enter. When forced to } \\
\text { move away, he immediately sat down next to control } 6 \text {, who was the first } \\
\text { participant he encountered. Because of symptoms, tests had been done. } \\
\text { The enzyme immunoassay gave a negative result; however, stool culture } \\
\text { showed non-toxigenic } C \text { difficile }\end{array}$ \\
\hline Control 7 & Inconclusive & Negative result & Yes & No apparent explanation \\
\hline \multicolumn{5}{|c|}{ False positivest: } \\
\hline Control 8 & Positive & Negative result & No & $\begin{array}{l}\text { Chlorine round, see comment for control } 2 \text {. Enzyme immunoassay gave } \\
\text { a negative result; however, stool culture showed non-toxigenic } C \text { difficile }\end{array}$ \\
\hline Control 9 & Positive & Not done & No & Participant offered the dog a cookie \\
\hline Control 10 & Positive & Not done & No & Participant beckoned the dog \\
\hline Control 11 & Positive & Negative result & No & Dog appeared distracted by urine on the floor from a broken catheter bag \\
\hline Control 12 & Positive & Negative result & No & $\begin{array}{l}\text { This participant had been treated for } C \text { difficile infection, diagnosed } 11 \\
\text { days previously. Since symptoms resolved on the day of the detection } \\
\text { round, the patient was included as a control. However, a week after the } \\
\text { round symptoms returned and retesting showed a relapse }\end{array}$ \\
\hline \multicolumn{5}{|c|}{ False negativesł: } \\
\hline Case 13 & Negative & Positive result & Yes & No clear explanation \\
\hline Case 14 & Negative & Positive result & Yes & $\begin{array}{l}\text { The enzyme immunoassay gave a positive result; culture was mistakenly } \\
\text { not done }\end{array}$ \\
\hline
\end{tabular}

*Enzyme immunoassay+toxigenic culture.

†Participants with negative laboratory results but positive dog response.

$\ddagger$ Participants with negative laboratory results but positive dog response. 


\section{Figures}

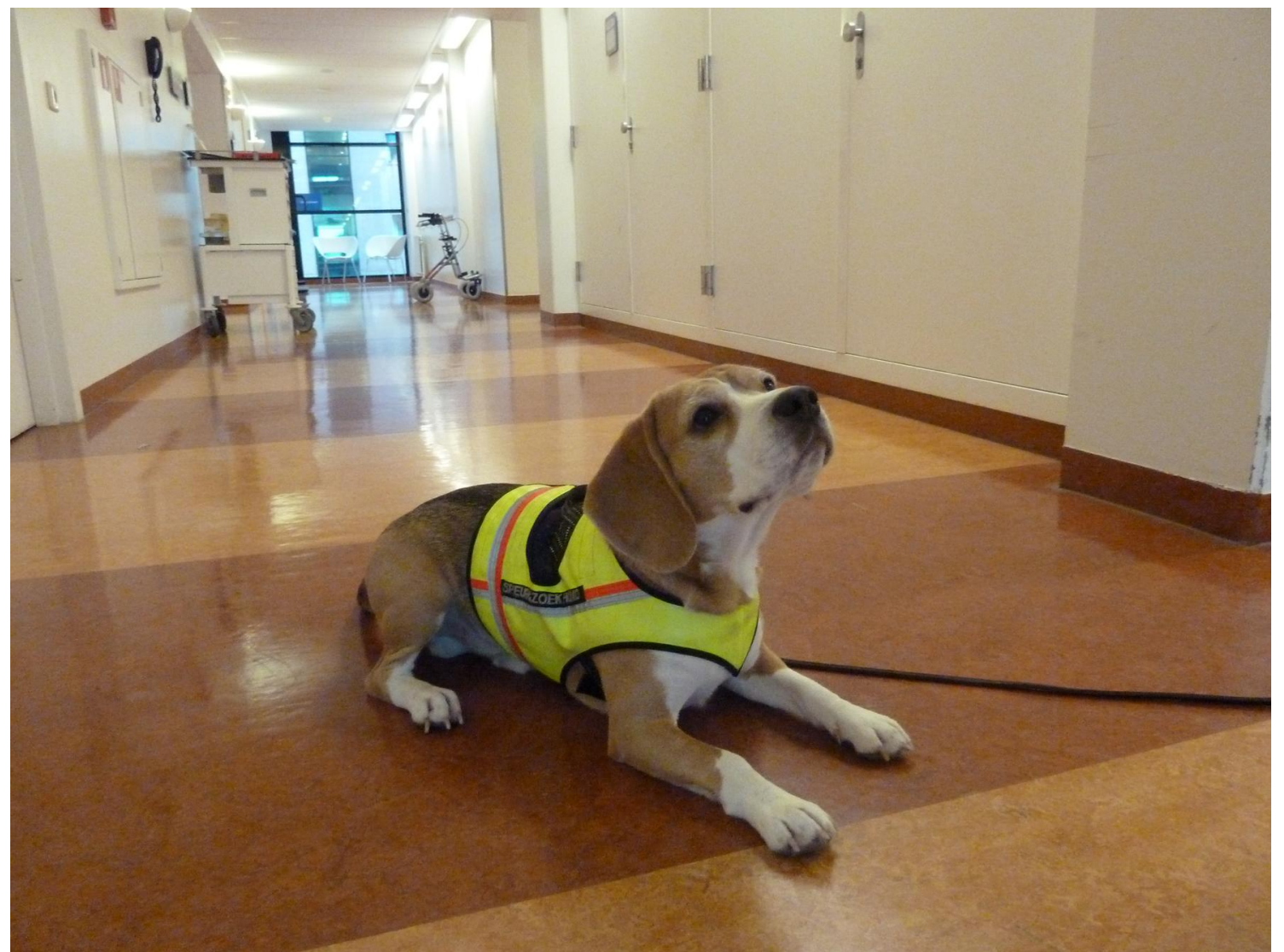

Fig 1 Detection dog on hospital ward

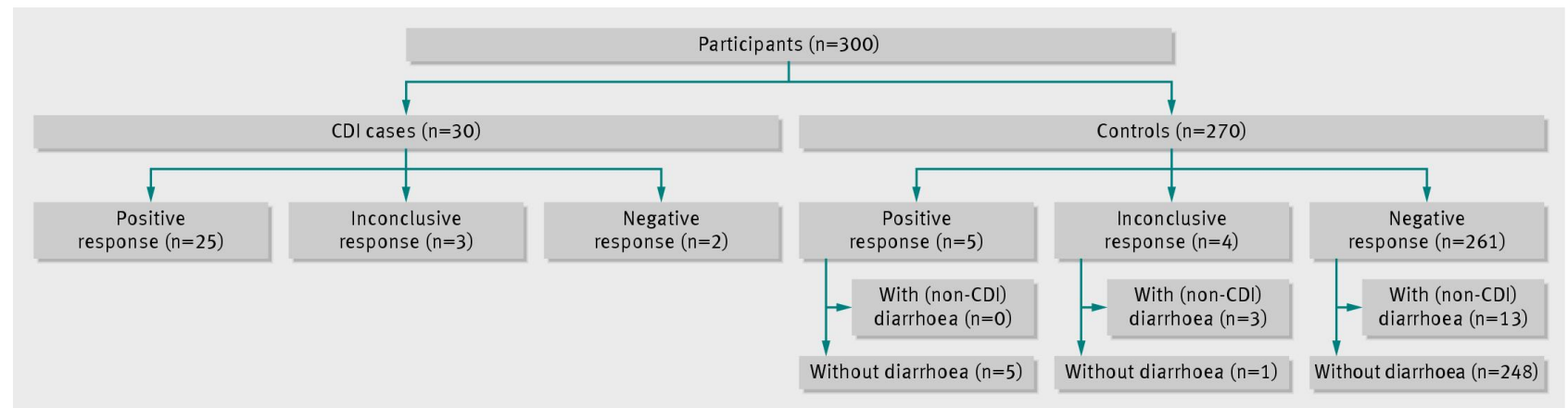

Fig 2 Diagnostic accuracy of dog for detecting Clostridium difficile infection (CDI) 